\title{
Changes of Thyroid Hormones and Their Binding Proteins during Plasma Exchange for Polyneuropathy in a Patient with Substituted Hypothyroidism due to Hashimoto's Thyroiditis
}

\author{
Stefan Jenni ${ }^{a} \quad$ Marcel Adler $^{b} \quad$ Simone Lanz ${ }^{a} \quad$ Behrouz Mansouri Taleghani ${ }^{b}$ \\ Emanuel R. Christ ${ }^{a}$ \\ a Department of Endocrinology, Diabetology and Clinical Nutrition, Bern University Hospital, Inselspital, Bern, Switzerland; \\ b University Clinic of Haematology and Central Haematology Laboratory, Bern University Hospital, Inselspital, Bern, Switzerland
}

\section{Keywords}

Thyroid hormones - Plasma exchange - Substitution . Hypothyroidism

\section{Summary}

Background: Pharmacodynamic studies and data concerning adaptation of thyroid substitution in patients with substituted hypothyroidism during plasma exchange (PE) is not available. Case Report: We measured $\mathrm{TSH}, \mathrm{fT}_{3}$ and $\mathrm{fT}_{4}$, total $\mathrm{T}_{4}$, thyroxin binding globulin (TBG), and albumin before and after 5 PE procedures in a 37-year-old women who underwent PE for a therapy-resistant polyneuropathy. Thyroxin was increased empirically by $8 \%$ resulting in a dose of $1.95 \mu \mathrm{g} / \mathrm{kg}$ per day. Results: Despite larger reductions of total $\mathrm{T}_{4}$ and TBG over a series of 5 PEs (40-50\% from baseline), only small reductions of $8 \%$ in $\mathrm{fT}_{3}$ and $\mathrm{fT}_{4}$ concentrations were documented with a concomittant increase in TSH level. Changes of $\mathrm{fT}_{4}, \mathrm{fT}_{3}$, and $\mathrm{TSH}$ remained within normal range. Conclusions: i) Despite a significant decrease in total thyroid hormone pool following $P E, \mathrm{fT}_{4}, \mathrm{fT}_{3}$, and TSH concentrations changed only slightly. ii) Based on this observation, a general increase in thyroid replacement therapy before PE cannot be recommended, but considered in case of a high normal TSH level.

(c) 2015 S. Karger GmbH, Freiburg

\section{Introduction}

Plasma exchange (PE) is the most commonly performed therapeutic apheresis procedure, according to data from international registries [1]. The basic premise of $\mathrm{PE}$ is that the removal of pathological or pathologically elevated substances will reduce further damage and may permit reversal of the pathologic process [2]. PE is recommended in several indications [3]. Primary hypothyrodism, mainly Hashimoto's autoimmune thyroiditis, is a frequent disorder with a prevalence of $0.3 \%$ for clinical and $4.3 \%$ for subclinical hypothyroidism [4]. Thyroxin $\left(\mathrm{T}_{4}\right)$ is mainly bound to thyroxin binding protein (TBG), albumin, and to a smaller extent to transthyretin. PE removes these binding proteins resulting in major changes of the total hormone pool $\left(\mathrm{TT}_{4}\right)$. In contrast to most other hormones, like insulin, cortisol and sex hormones that are rapidly cleared from the circulation and resecreted again if needed, $\mathrm{T}_{4}$ has an especially prolonged half-life time of around 7 days, a small fraction of free hormone (free thyroxine $\left(\mathrm{fT}_{4}\right)$ around $0.03 \%$ ) and a quite stable plasma level throughout the day. In addition to the shift from bound to freely available thyroid hormone, the pituitary-thyroid axis is thought to compensate for a PE-induced reduction in $\mathrm{TT}_{4}$ in a physiological condition [5]. However, pharmacodynamic studies or data about an adaptation of a given thyroid replacement therapy in view of a PE in a patient with hypothyroidism are currently not available.

We, therefore, aimed at investigating the effect of PE on thyroid hormone metabolism in a patient with therapy-resistant polyneuropathy who was treated for primary hypothyroidism (Hashimoto's thyroiditis). 
Table 1. Immediate and steady-state changes of thyroid function tests and related binding proteins during 5 courses of $\mathrm{PE}$ and their related changes

\begin{tabular}{|c|c|c|c|c|}
\hline & \multicolumn{2}{|c|}{ Immediate changes ${ }^{\star}(\mathrm{n}=5)$} & \multicolumn{2}{|c|}{ Steady-state changes $*(n=4)$} \\
\hline & absolute & relative & absolute & relative \\
\hline $\mathrm{TSH}, \mathrm{mU} / \mathrm{l}$ & $-0.37 \pm 0.30$ & $-45.9 \pm 6.3 \%$ & $0.41 \pm 0.48$ & $37.8 \pm 25.0 \%$ \\
\hline $\mathrm{fT}_{4}, \mathrm{pmol} / \mathrm{l}$ & $-1.4 \pm 1.0$ & $-7.9 \pm 5.7 \%$ & $-0.75 \pm 1.6$ & $-4.5 \pm 9.0 \%$ \\
\hline $\mathrm{TT}_{4}, \mathrm{nmol} / \mathrm{l}$ & $-36 \pm 8.3$ & $-45.4 \pm 6.6 \%$ & $-4.5 \pm 10.5$ & $-6.1 \pm 14.4 \%$ \\
\hline $\mathrm{fT}_{4} / \mathrm{TT}_{4}, \%$ & $0.0183 \pm 0.0082$ & $80.3 \pm 34.4 \%$ & $0.00025 \pm 0.0024$ & $0.76 \pm 10.1 \%$ \\
\hline $\mathrm{fT}_{3}, \mathrm{pmol} / \mathrm{l}$ & $-0.29 \pm 0.12$ & $-6.9 \pm 2.1 \%$ & $-0.33 \pm 0.08$ & $-8.5 \pm 2.1 \%$ \\
\hline TBG, mg/l & $-11.0 \pm 2.35$ & $-62.0 \pm 7.4 \%$ & $-0.25 \pm 4.35$ & $-4.0 \pm 28.4 \%$ \\
\hline Albumin, g/l & $-0.2 \pm 3.1$ & $-0.3 \pm 8.2 \%$ & $0.75 \pm 1.5$ & $1.9 \pm 3.8 \%$ \\
\hline
\end{tabular}

\section{Case Report}

An informed consent form was signed by the patient approving the use of material or information for scientific purposes.

We present the case of a 37-year-old woman with a severe painful peripheral polyneuropathy for 3 years. The etiology could not be determined although a chronic inflammatory demyelinizing polyneuropathy was suspected. An initial treatment with oral steroids did not relieve symptoms. Also a therapy with intravenous immunoglobulins did not result in satisfactory symptomatic relief. Due to persistent symptoms a series of PEs was planned. The patient was known for primary hypothyroidism due to Hashimoto's thyroiditis since the age of 10 with documented elevated TPO antibodies. She was on stable thyroid replacement therapy.

\section{Methods}

\section{PE Procedures}

The mobile centrifugal apheresis system Spectra Optia (TerumoBCT, Lakewood, CO, USA) was used. Procedures were conducted by qualified nurses supervised by a trained physician. Within 14 days a total of 5 PEs were performed every 2-5 days. Each time the 1.2-fold of the patient's own plasma volume was replaced using a $5 \%$ albumin solution (CSL Behring AG, Bern, Switzerland) containing at least $96 \%$ albumin according to the manufacturer and physiologic saline (Sintetica SA, Couvet, Switzerland) in a ratio of 2:1.

We used citrate (ACD-A, Bichsel SA, Interlaken, Switzerland) as anticoagulant, following the manufacturers' instructions. Routinely, an intravenous continuous infusion of calcium chloride (Calcium-Sandoz 10\%, Sandoz AG, Risch, Switzerland) throughout the PE was performed. The initial infusion rate corresponded to the administration of median $0.25 \mathrm{mg}$ of ionized calcium $\left(\mathrm{Ca}^{2+}\right)$ per milliliter of ACD-A (0.53 $\mathrm{mmol} \mathrm{Ca}{ }^{2+} / 10 \mathrm{mmol}$ citrate) [6].

\section{Substitution}

The patient presented with a thyroid-stimulating hormone (TSH) level of $3.36 \mathrm{mU} / \mathrm{l}$ at baseline under a stable levothyroxine dose of $129 \mu \mathrm{g} / \mathrm{day}$ $(1.81 \mu \mathrm{g} / \mathrm{kg} /$ day). As we anticipated a loss during PE, we empirically increased the substitution dose to $139 \mu \mathrm{g} /$ day $(+8 \% ; 1.95 \mu \mathrm{g} / \mathrm{kg} /$ day) 3 weeks before the first PE. After the last PE the patient returned to her usual dose.

\section{Laboratory Samples}

Plasma samples were drawn before and immediately after PE. TSH, $\mathrm{fT}_{3}$ (free liothyronine), and $\mathrm{fT}_{4}$ were determined using electro-chemiluminescence immunoassay (Modular E170; F. Hoffmann-La Roche AG, Basel, Switzerland), and for albumin the bromcresol purple method was used (Modular P800; F. Hoffmann-La Roche AG) using routine laboratory. $\mathrm{TT}_{4}$ and $\mathrm{TBG}$ was determined using the Chemiluminescent Microparticle Immunoassay, Architect
(Abbott Laboratories, Abbott Park, IL, USA) and a radio immunoassay, respectively, in an external laboratory (Laboratoire chimie clinique, Endocrinologie, Centre hospitalier universitaire Vaudois, Lausanne).

\section{Statistical Analysis}

Due to the small sample size only descriptive methods (arithmetic mean) for changes are reported. We calculated immediate differences (value post-PE minus value pre-PE) of all 5 procedures (both in absolute and relative terms, the mean \pm SD of 5 plasmaphereses is given). The relative immediate differences reflect real removal and were calculated as: $100-(($ value post-PE $\times 100)$ / value pre-PE). Assuming a one-compartment model, a predicted removal can be calculated as: $100-\left(\left(\left(\right.\right.\right.$ value pre- $\left.\left.\mathrm{PE} \times \mathrm{e}^{-\mathrm{x}}\right) \times 100\right) /$ value pre-PE), where $\mathrm{x}$ is the plasma exchanged [7].

A new steady-state condition was assumed to be reflected by the values measured before each PE. We, therefore, calculated differences from the beginning of one PE to the next. The mean $( \pm$ SD) of 4 steady-state differences in absolute and relative terms is reported. In order to determine whether or not the number of PE impacts on the different parameters of thyroid hormone metabolism the values before the first and the last procedure were considered.

\section{Results}

\section{Clinical Course}

Clinically, a focused history did not reveal any symptoms of a dysthyroid condition throughout the procedures. In electroneurography studies, minor improvements of polyneuropathy parameters could be detected. However, the patient reported no subjective improvement after the 5 PEs. The treatment was, therefore, discontinued after 5 procedures.

\section{Laboratory Values}

The minor dose increase of thyroid replacement therapy 3 weeks before the first PE resulted in a reduction of TSH levels slightly below the lowest limit of normal range $(0.22 \mathrm{mU} / \mathrm{l}$; normal range $0.27-4.5$ $\mathrm{mU} / \mathrm{l})$. The changes of the thyroid function tests and the related binding proteins are shown in figure 1 . Before the first and the last $\mathrm{PE}$ the TSH increased from 0.22 to $1.87 \mathrm{mU} / \mathrm{l}, \mathrm{fT}_{4}$ decreased from 19.7 to $16.7 \mathrm{pmol} / \mathrm{l}$ and $\mathrm{fT}_{3}$ from 4.78 to $3.45 \mathrm{pmol} / \mathrm{l}$ (all within normal range). $\mathrm{TT}_{4}$ was $95 \mathrm{nmol} / \mathrm{l}$ before the $1 \mathrm{st}$ and $77 \mathrm{nmol} / \mathrm{l}$ before the 5th PE (normal range 60-150 nmol/l). TBG completely recovered after each PE. Albumin remained constant, as it was substituted. 
Fig. 1. Thyroid function tests and related binding proteins across 5 PEs in a patient with substituted primary hypothyroidism. Dashed lines indicate normal reference values. Full circles indicate values before and hollow circles values post plasma exchange.
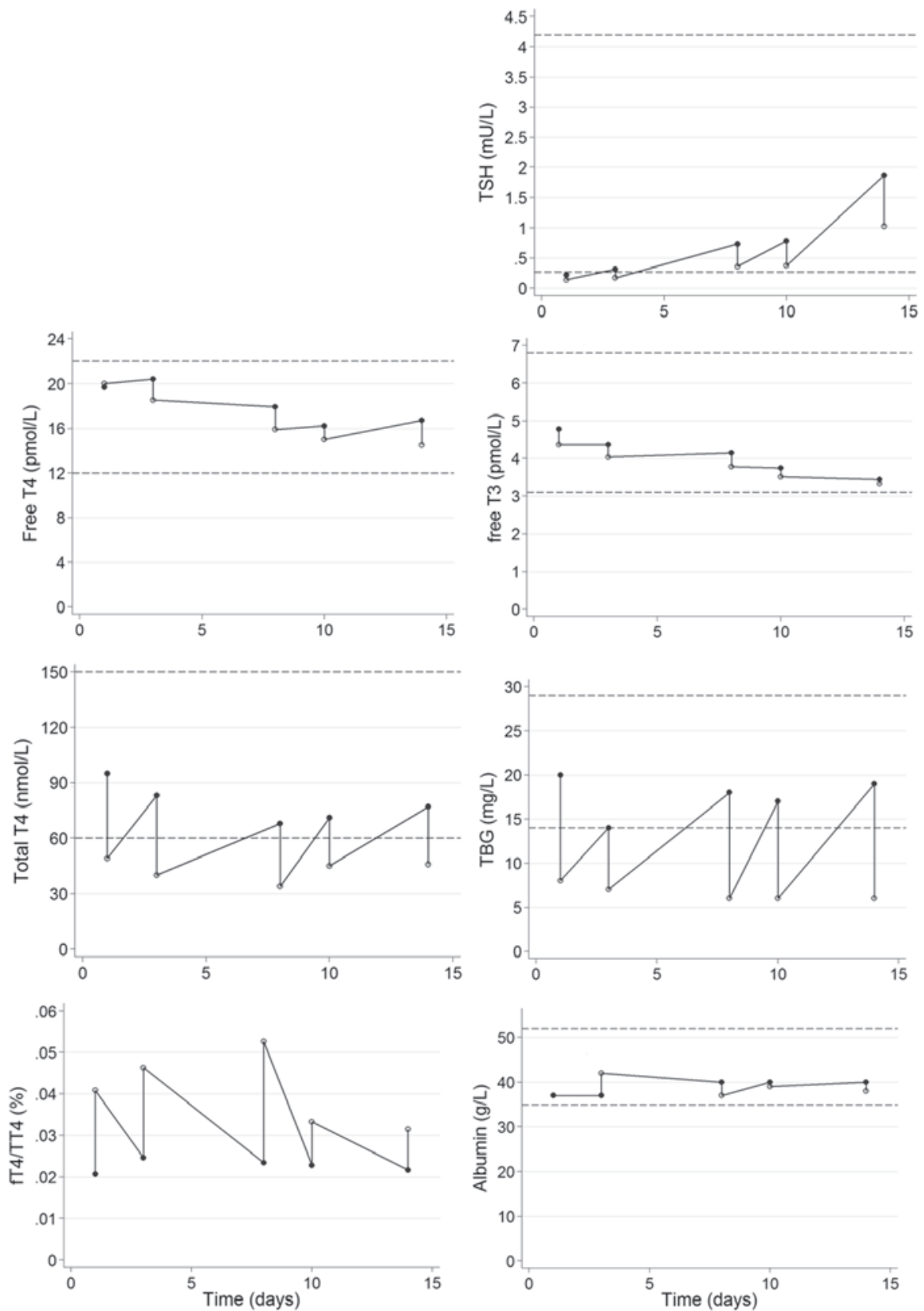

In table 1 the PE-induced immediate changes are compared with steady-state changes. Assuming a one-compartment model, a removal of $69.9 \%$ would be expected. Total $\mathrm{T}_{4}$ fell on average by 45 $\pm 6.3 \%$ during each PE, reflecting the reduction of TBG by $62 \pm$ $7.4 \%$. The relative decrease in TSH concentrations was similar $(45.9 \pm 6.3 \%)$. The free hormones were only reduced by $7.9 \pm 5.7 \%$ for $\mathrm{fT}_{4}$, and by $-6.9 \pm 2.1 \%$ for $\mathrm{fT}_{3}$. Consequently, the ratio of $\mathrm{fT}_{4}$ to $\mathrm{TT}_{4}$ increased significantly.

In the steady state the TBG changes were largely abolished (-6.1 $\pm 14.4 \%)$, as were TT4 changes $(-4.5 \pm 9.0 \%)$, but for TSH an increase was found $(37.8 \pm 25.0 \%)$. The reductions in free hormone concentrations were comparable to the immediate changes $(-4.5 \pm$ $9.0 \%$ for $\mathrm{fT}_{4},-8.5 \pm 2.1 \%$ for $\left.\mathrm{fT}_{3}\right)$. The free-to-bound ratio remained constant over the course of the PE.

Thyroid Hormone Substitution during Plasma Exchange

\section{Discussion}

The main results from this report are three-fold: i) A PE induces an immediate decrease in $\mathrm{TT}_{4}$ and $\mathrm{TBG}$ below normal range that recovers and stabilizes in the lower half of normal range after 5 consecutive procedures. ii) Five PE within 14 days resulted in a steady decrease in $\mathrm{fT}_{4}$ and $\mathrm{fT}_{3}$ concentrations and an increase in TSH levels, albeit all within normal range. iii) Based on these findings, an increase in the dose of thyroid replacement therapy before $\mathrm{PE}$ cannot be generally recommended. A thyroid replacement dose inducing a TSH in the lower normal range can be considered before starting the first PE.

Acute changes of thyroid parameters included a decrease in $\mathrm{TT}_{4}$ pool in parallel with a decrease in TBG. Interestingly, the values 
immediately after the PE were lower than the normal range. This is not surprising since $99.97 \%$ of $\mathrm{T}_{4}$ is bound, with about three quarters bound to TBG. In fact, $\mathrm{TT}_{4}$ relates to the 3,400-fold of the free hormone pool. The removal of TBG $(-62 \%)$ was close to the expected value $(-69.9 \%)$, whereas $\mathrm{TT}_{4}$ was somewhat lower $(-45 \%)$. The lower than expected values could be due to TBG and $\mathrm{T}_{4}$ included in the albumin preparation, a similar effect has been shown for haptoglobin [7]. In contrast, $\mathrm{fT}_{4}$, and $\mathrm{fT}_{3}$ decreased only slightly - far off from a logarithmical removal - indicating a shift from the bound $\mathrm{T}_{4}$ pool to the free $\mathrm{T}_{4}$ fraction. This is reflected by an increase in the $\mathrm{fT}_{4} / \mathrm{TT}_{4}$ ratio and may explain the lack of significant clinical symptoms and signs throughout the procedures. There is a tendency for an increase in $\mathrm{TT}_{4}$ during the last $\mathrm{PE}$, while $\mathrm{TBG}$ remained stable throughout the procedures. Apart from a possible increase in production rate of TBG, possibly there was also a reverse flow of extravascular $\mathrm{T}_{4}$ as shown in a rabbit model [8].

$\mathrm{PE}$ has been described as an effective method of last therapeutic resort for therapy-resistant thyrotoxicosis [9] and thyroxine intoxication [10]. In both situations substantially higher $\mathrm{fT}_{4}$ levels are reported based on an enhanced endogenous production or intake. Due to the ongoing endogenous production, PE is rather considered a temporal relief therapy before definitive surgery. Taking in account the current observation of only small reductions in $\mathrm{fT}_{4}$ and $\mathrm{fT}_{3}$ and only insignificant reductions of $\mathrm{fT}_{4}$ in one retrospective study [9] following PE, the utility of such a procedure has to be questioned, although it may be a life-saving procedure for thyroid storm in the case of intoxication [11].

There is a steady decrease in $\mathrm{fT}_{4}$ and $\mathrm{fT}_{3}$ with a concomitant increase in TSH after 5 procedures, albeit within normal range. This indicates that - in contrast to $\mathrm{TT}_{4}$ and TGB - the compensatory mechanisms are not capable to guarantee completely stable $\mathrm{fT}_{4}$ and $\mathrm{fT}_{3}$ levels throughout several PEs, and more than 5 PEs may eventually result in a hypothyroid condition. Compensatory mechanisms include the shift from bound to free thyroid hormone pool and/or TSH-stimulated production of thyroid hormone. The current study was not designed to differentiate between these mechanisms. However, the observation of an increase in the $\mathrm{fT}_{4} / \mathrm{TT}_{4}$ ratio and TSH levels suggest that both mechanisms were probably involved. Interestingly, in euthyroid patients without Hashimoto's thyroiditis, PE was reported not to result in significant changes of free hormones despite the fact that similar changes for $\mathrm{TT}_{4}$ and TBG were observed [5].

\section{Limitations}

The main limitation of this report is that it is restricted to one subject with only 5 instances of PE. Clinically more relevant changes may become apparent in patients with more than 5 procedures.

\section{Strength}

The setting has been highly standardized with a sufficient temporal resolution and additional measurement of clinically now rarely used parameters.

\section{Recommendation}

Based on these data it appears reasonable to aim for a TSH level in the lower normal range before plasma exchange is initiated. At least for 5 consecutive PEs, a general increase in thyroid substitution dose cannot be recommended. However, if more than 5 procedures are planned in a series, an adaption of thyroid hormone replacement dose may be considered. After plasma exchange the original substitution regimen can be re-adopted. Frequent measurements of $\mathrm{TSH}, \mathrm{fT}_{3}$, and $\mathrm{fT}_{4}$ are mandatory to further adapt the substitution dose.

\section{Conclusion}

Despite a significant decrease in $\mathrm{TT}_{4}$ following plasma exchange, $\mathrm{fT}_{4}, \mathrm{fT}_{3}$ and $\mathrm{TSH}$ concentrations changed only slightly.

Based on this observation, a general increase in thyroid replacement therapy before plasma exchange cannot be recommended.

An increase in substitution dose can be considered in case of a high normal TSH level.

Plasma exchange in patients with substituted hypothyroidism may serve as a system's pertubation tool to investigate the physiology of thyroid hormones and their binding proteins. Results from such observations may help quantify expected losses for future patients.

\section{Disclosure Statement}

The authors declare that they do not have conflicts of interest.

\section{References}

1 Stegmayr B, Ptak J, Wikstrom B, Berlin G, Axelsson CG, Griskevicius A, Centoni P, Liumbruno G, Molfettini P, Audzijoniene J, Mokvist K, Sojka BN, Norda R, Knutson F, Ramlow W, Blaha M, Witt V, Evergren M, Tomaz J: World apheresis registry 2003-2007 data. Transfus Apher Sci 2008;39:247-254.

2 Reeves HM, Winters JL: The mechanisms of action of plasma exchange. Br J Haematol 2014;164:342-351.
3 Schwartz J, Winters JL, Padmanabhan A, Balogun RA, Delaney M, Linenberger ML, Szczepiorkowski ZM, Williams ME, Wu Y, Shaz BH: Guidelines on the use of therapeutic apheresis in clinical practice-evidencebased approach from the Writing Committee of the American Society for Apheresis: the sixth special issue. J Clin Apher 2013;28:145-284.
4 Hollowell JG, Staehling NW, Flanders WD, Hannon WH, Gunter EW, Spencer CA, Braverman LE: Serum TSH, T(4), and thyroid antibodies in the United States population (1988 to 1994): National Health and Nutrition Examination Survey (Nhanes Iii). Journal of Clin Endocrinol Metab 2002;87:489-499.

5 Sakata S, Komaki T, Kojima N, Miura K: The effect of double filtration plasmapheresis on thyroid hormone economy and thyroid function. Jap J Med 1986;25: 246-249. 
6 Buchta C, Macher M, Bieglmayer C, Hocker P, Dettke $\mathrm{M}$ : Reduction of adverse citrate reactions during autologous large-volume PBPC apheresis by continuous infusion of calcium-gluconate. Transfusion 2003;43: 1615-1621.

7 Cid J, Elena M, Diekmann F, Lozano M: Haptoglobin is present in albumin used as a replacement solution for plasma exchange. Transfusion 2013;53:757-760.
Wahl R, Schmidberger H, Fessler E, Heinzel W, Schenzle D, Bohner J, Kallee E: Effects of human thyroxine-binding globulin and prealbumin on the reverse flow of thyroid hormones from extravascular space into the bloodstream in rabbits. Endocrinology 1989;124:1428-1437. 9 Ezer A, Caliskan K, Parlakgumus A, Belli S, Kozanoglu I, Yildirim S: Preoperative therapeutic plasma exchange in patients with thyrotoxicosis. Journal Clin Apher 2009;24:111-114.
10 Binimelis J, Bassas L, Marruecos L, Rodriguez J, Domingo ML, Madoz P, Armengol S, Mangues MA, de Leiva A: Massive thyroxine intoxication: evaluation of plasma extraction. Intensive Care Med 1987;13:33-38.

11 Jha S, Waghdhare S, Reddi R, Bhattacharya P: Thyroid storm due to inappropriate administration of a compounded thyroid hormone preparation successfully treated with plasmapheresis. Thyroid 2012;22:12831286. 